\title{
Measurement of $B\left(D_{s}^{+} \rightarrow \mu^{+} \nu_{\mu}\right) / B\left(D_{s}^{+} \rightarrow \phi \mu^{+} \nu_{\mu}\right)$ and Determination of the Decay Constant $f_{D_{s}}$
}

\author{
Fermilab E653 Collaboration \\ K. $\operatorname{Kodama}^{(1)}$, S. Torikai ${ }^{(1)}$, N. Ushida(1), A. Mokhtarani ${ }^{(2),(a)}$, V.S. Paolone ${ }^{(2)}$, \\ J.T. Volk ${ }^{(2),(a)}$, J.O. Wilcox ${ }^{(2),(b)}$, P.M. Yager ${ }^{(2)}$, R.M. Edelstein ${ }^{(3)}$, A.P. Freyberger ${ }^{(3),(c)}$, \\ D.B. Gibaut ${ }^{(3),(d)}$, R.J. Lipton ${ }^{(3),(a)}$, W.R. Nichols ${ }^{(3),(e)}$, D.M. Potter ${ }^{(3)}$, J.S. Russ ${ }^{(3)}$, \\ C. Zhang ${ }^{(3)}$, Y. Zhang( ${ }^{(3),(f)}$, H.I. Jang ${ }^{(4)}$, J.Y. Kim ${ }^{(4)}$, B.R. Baller ${ }^{(5)}$, R.J. Stefanski ${ }^{(5)}$, \\ K. Nakazawa ${ }^{(6)}$, S.H. Chung ${ }^{(7)}$, M.S. Park ${ }^{(7)}$, I.G. Park ${ }^{(7)}$, J.S. Song ${ }^{(7)}$, C.S. Yoon ${ }^{(7)}$, \\ M. Aryal ${ }^{(8)}$, N.W. Reay ${ }^{(8)}$, R.A. Sidwell ${ }^{(8)}$, N.R. Stanton ${ }^{(8)}$, S. Yoshida $^{(8)}$, M. Chikawa ${ }^{(9)}$,

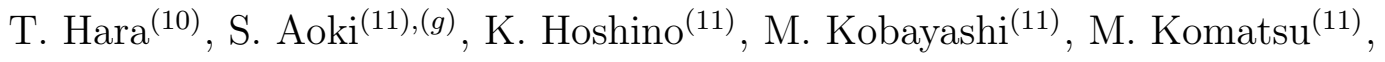 \\ M. Miyanishi ${ }^{(11)}$, M. Nakamura(11), S. Nakanishi(11), K. Niwa(11), M. Nomura(11),

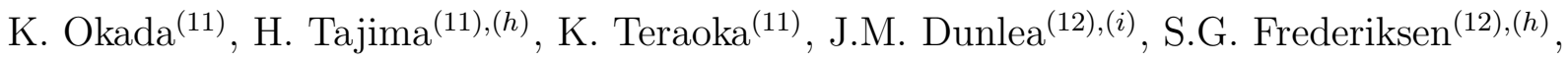

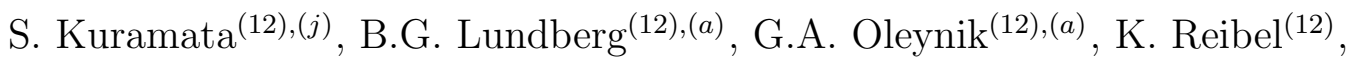 \\ G.R. Kalbfleisch ${ }^{(13)}$, P. Skubic ${ }^{(13)}$, J.M. Snow ${ }^{(13)}$, S.E. Willis ${ }^{(13),(k)}$, K. Nakamura ${ }^{(14)}$,

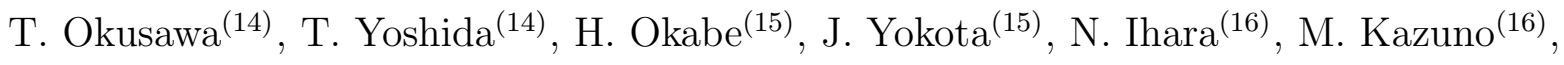 \\ T. Koya ${ }^{(16)}$, E. Niu ${ }^{(16),(l)}$, S. Ogawa ${ }^{(16)}$, H. Shibuya ${ }^{(16)}$, S. Watanabe ${ }^{(16),(m)}$, N. Yasuda ${ }^{(16)}$,

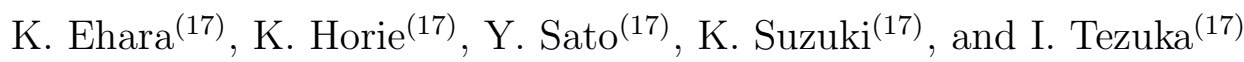

\begin{abstract}
We have observed $23.2 \pm 6.0_{-0.9}^{+1.0}$ purely-leptonic decays of $D_{s}^{+} \rightarrow \mu^{+} \nu_{\mu}$ from a sample of muonic one prong decay events detected in the emulsion target of Fermilab experiment E653. Using the $D_{s}^{+} \rightarrow \phi \mu^{+} \nu_{\mu}$ yield measured previously in this experiment, we obtain $B\left(D_{s}^{+} \rightarrow \mu^{+} \nu_{\mu}\right) / B\left(D_{s}^{+} \rightarrow \phi \mu^{+} \nu_{\mu}\right)=0.16 \pm 0.06 \pm 0.03$. In addition, we extract the decay constant $f_{D_{s}}=194 \pm 35 \pm 20 \pm 14 \mathrm{MeV}$.

1) Aichi University of Education, Kariya 448, JAPAN

2) University of California (Davis), Davis, CA 95616, USA
\end{abstract}


3) Carnegie-Mellon University, Pittsburgh, PA 15213, USA

4) Chonnam National University, Kwangju 500-757, KOREA

5) Fermi National Accelerator Laboratory, Batavia, IL 60510, USA

6) Gifu University, Gifu 501-11, JAPAN

7) Gyeongsang National University, Jinju 660-300, KOREA

8) Kansas State University, Manhattan, KS 66506, USA

9) Kinki University, Higashi-Osaka 577, JAPAN

10) Kobe University, Kobe 657, JAPAN

11) Nagoya University, Nagoya 464, JAPAN

12) The Ohio State University, Columbus, OH 43210, USA

13) University of Oklahoma, Norman, OK 73019, USA

14) Osaka City University, Osaka 558, JAPAN

15) Science Education Institute of Osaka Prefecture, Osaka 558, JAPAN

16) Toho University, Funabashi 274, JAPAN

17) Utsunomiya University, Utsunomiya 321, JAPAN

${ }^{a}$ Fermilab, Batavia, IL 60510, USA.

${ }^{b}$ Northeastern University, Boston, MA 02115, USA.

${ }^{c}$ CEBAF, 12000 Jefferson Avenue Newport News, VA 23606, USA.

${ }^{d}$ Virginia Polytechnic Institute and State University, Blacksburg, VA 24060, USA.

${ }^{e}$ Westinghouse Electric Corp., Pittsburgh, PA 15230, USA.

${ }^{f}$ Pennsylvania State University, University Park, PA 16802, USA.

${ }^{g}$ Kobe University, Kobe 657, JAPAN.

${ }^{h}$ University of Tokyo 7-3-1 Hongo, Bunkyou-ku, Tokyo 113 JAPAN.

${ }^{i}$ University of Rochester, Rochester, NY 14627, USA.

${ }^{j}$ Hirosaki University, Hirosaki 036, JAPAN.

${ }^{k}$ Northern Illinois University, DeKalb, IL 60115, USA.

${ }^{l}$ CERN, CH-1211, Geneva, SWITZERLAND.

${ }^{m}$ University of Wisconsin, Madison, WI 53706, USA. 
Leptonic decay of charged pseudoscalar mesons is described by the annihilation of constituent quark and antiquark into a virtual $W$ boson. The rate for this process is proportional to the square of the pseudoscalar decay constant $f$, which measures the overlap of quark and antiquark at zero separation. The constant $f$ gives absolute normalizations of numerous heavy-flavor transitions, including mixing, semi-leptonic and non-leptonic decays. Hence it is highly desirable to determine the pseudoscalar decay constant more precisely in charm and $B$ physics. A measurement of a purely-leptonic decay branching ratio, unlike semi-leptonic or non-leptonic decays, is the most reliable way to extract the pseudoscalar decay constant, because it does not involve any QCD corrections.

There are three purely-leptonic decay modes for the $D_{s}^{+}$(charge conjugate modes are implied throughout this paper): electronic, muonic and tauonic. Among them, the muonic decay is the most accessible mode to determine the decay constant $f_{D_{s}}$. The electronic decay is helicity suppressed by five orders of magnitude with respect to the muonic one. While the tauonic decay branching ratio is expected to be an order of magnitude larger than the muonic decay, the $\mathrm{Q}$ value for $D_{s}^{+} \rightarrow \tau^{+} \nu_{\tau}$ is small and hence reconstruction of this mode is extremely difficult because of the small decay angle in the lab frame.

The first measurement of the pseudoscalar decay constant of the charmed strange meson, $f_{D_{s}}$, was carried out by the WA75 group in 1993 [电, using a transverse momentum spectrum of muons from $D_{s}^{+}$leptonic decays observed in an emulsion target. They obtained a value of $f_{D_{s}}=(232 \pm 45 \pm 20 \pm 48) \mathrm{MeV}$. CLEO [2] and BES [i] also reported recent measurements of $f_{D_{s}}$, and ARGUS 幽 obtained $f_{D_{s}}$ by model dependent calculation in 1992. All of these results, however, suffer from large statistical and systematic errors, and hence the present measured value of $f_{D_{s}}$ is still not determined well enough to discriminate between different theoretical models. In this paper, we report an independent measurement of $f_{D_{s}}$ from an analysis of the data from Fermilab experiment E653.

The Fermilab emulsion-hybrid experiment E653 was designed to study production and decay of heavy flavor particles by the direct observation of decay vertices in the emulsion. Data for this analysis were taken in a $600 \mathrm{GeV} / \mathrm{c} \pi^{-}$beam during the second run of Fermilab 
E653. The trigger required both a beam particle to interact in the target and a muon to penetrate $3,900 \mathrm{~g} / \mathrm{cm}^{2}$ of absorber. This muon trigger provided an enriched sample of semi-muonic decays of heavy flavor particles. A total of $8.2 \times 10^{6}$ events, corresponding to $2.5 \times 10^{8}$ interactions, were recorded during this run. Since the details of this experiment were described in another paper [ [0 $]$ ], only a brief description of each detector element is given here.

The detector consisted of the emulsion target, tracking detector, spectrometer, and muon detection system. Because of its unsurpassed spatial resolution, nuclear emulsion was chosen as an active target, and it was used both as a primary interaction target and as a decay vertex detector. The electronic detectors downstream of the target select events for the emulsion scanning and predict the interaction vertices and each track position in the emulsion. Tracks from interactions and decays in the emulsion were measured in the vertex silicon strip detectors (VSSDs). The VSSDs consisted of 18 planes arranged in 6 groups of three readout coordinates, and the center part of the detector achieved a resolution transverse to the beam of $8.8 \mu \mathrm{m}$. This detector also served as the upstream arm of the spectrometer. The spectrometer gave a momentum resolution of $\sigma_{p} / p=\sqrt{(0.01)^{2}+(0.00023 p)^{2}}(p$ in GeV/c). The most down stream element of the E653 detector array was the muon identification system, which remeasured the direction and momenta of muon candidates after the absorber with drift chambers and an iron toroid.

To enrich the relative yield of events with heavy flavor particle decays and to reduce the emulsion scanning load, the transverse momentum of the triggered muon with respect to the beam axis, $P_{T \mu \text { Beam }}$, was required to be greater than $0.8 G e V / c$. In total, 94,000 events passed this criterion from a initial sample of $8.2 \times 10^{6}$ events. This reduced sample contained a significant number of background events from feedthrough muons. Pions or kaons could be misidentified as muons because they decayed muonically in flight with small angle deflections or from punch through in the iron absorber. These events were rejected using the method described below.

In the emulsion analysis, the primary interaction vertices were visually located in the 
emulsion by a semi-automatic scanning microscope system using position predictions from the VSSDs. The efficiency for finding primary vertices was determined to be greater than $99 \%$. If the angle of an emulsion track from the primary vertex matched to within $1 \mathrm{mrad}$ of the reconstructed muon track, the muon was identified as a feedthrough muon and the event was rejected. For the remaining events, a more detailed emulsion scanning technique was employed to find the origin of the muon.

Different decay search methods were applied for charged and neutral decay vertices. To find charged decays, all emulsion tracks observed at the primary vertex which did not match to any track reconstructed by the VSSDs were followed towards the most downstream emulsion plates. To find neutral decays, the tracks reconstructed by the VSSDs with no corresponding emulsion track observed at the primary vertex, were located at the most downstream plate of the emulsion target. Once found, these tracks were followed upstream through the emulsion stack to their origin within the emulsion target to detect neutral decays. The detailed event location and vertex search methods are described elsewhere [6].

As a result of the above analysis, 1,193 charmed particle candidates were identified: 565 KINK (charged one prong) decays, $536 \mathrm{VEE}$ (neutral two prong) decays, and 92 three or four

prong decays. Further cuts on the decay length $(>500 \mu m)$ and the transverse momentum of the muon with respect to the parent particle direction $\left(P_{T \mu}>0.28 \mathrm{GeV} / \mathrm{c}\right)$ were applied to the KINK and VEE decays to improve position resolution measurement and to reject strange particle decays. Only VEEs with their vertices found in the emulsion were retained to avoid the large $P_{T \mu}$ error due to the intrinsically larger position errors of the VSSDs. The offline selection criteria and cuts are summarized in Table 1 .

\section{TABLE 1}

Figure 1 shows the $P_{T \mu}$ distributions for $531 \mathrm{KINKs}$ and 276 VEEs which survived the above selection criteria. An excess of 19 events can be seen in the region $\left(P_{T \mu}>0.865 \mathrm{GeV} / \mathrm{c}\right)$ exceeding the kinematic limit of the $D^{+} \rightarrow \bar{K}^{0} \mu^{+} \nu_{\mu}$ decay for the KINK events, while no such signal is seen for the VEE events. These large $P_{T \mu}$ events can be interpreted as the 
fully leptonic decay $D_{s}^{+} \rightarrow \mu^{+} \nu_{\mu}$.

In addition to $D_{s}^{+} \rightarrow \mu^{+} \nu_{\mu}$ this sample includes background events. The largest source of background is the muon from the decay $D^{+} \rightarrow \mu^{+} \nu_{\mu}$. The inclusive production cross section for $D^{+}$is a factor of two larger than $D_{s}$. However, the branching fraction for this decay is considerably smaller than $D_{s}^{+} \rightarrow \mu^{+} \nu_{\mu}$, because it is suppressed by the ratio of CKM matrix-element, $\left|V_{c d}\right|^{2} /\left|V_{c s}\right|^{2}$. A muon from the cascade decay, $D_{s}^{+} \rightarrow \tau^{+} \nu_{\tau}$; $\tau^{+} \rightarrow \mu^{+} \nu_{\mu} \overline{\nu_{\tau}}$ is another source of background. Since the decay angle of the first decay point is extremely small $(\approx 1-2 \mathrm{mrad})$, it is often not detectable by our ordinary emulsion scanning method [6]. Therefore, only the second decay $\tau^{+} \rightarrow \mu^{+} \nu_{\mu} \overline{\nu_{\tau}}$ is observable and therefore will appear in the emulsion as a muonic KINK. A final possible background is a feedthrough muon from the daughter track of a hadronic charm decay.

We used a Monte Carlo simulation to estimate the total number of background events. Charged and neutral charmed particles were generated, using the production distribution parametrization $d^{2} \sigma / d x_{F} d P_{T}^{2} \propto\left(1-\left|x_{F}\right|\right)^{n} \cdot \exp \left(-b P_{T}^{2}\right)$ with $n=4.25 \pm 0.24 \pm 0.23$ and $b=0.76 \pm 0.03 \pm 0.03(\mathrm{GeV} / \mathrm{c})^{-2}$ [目]. The relative numbers of $D^{+}, D^{0}$ and $D_{s}^{+}$mesons were generated according to the the cross section values measured in this experiment [8, , , 18]. The baryon $\Lambda_{c}^{+}$was generated using the cross section value obtained from other experiments [0]]. The cross section numbers employed in the Monte Carlo are summarized in Table 2. Decays of charmed particles were simulated by JETSET 7.4 with known branching fractions [8], except that the ratio $f_{D^{+}} / f_{D_{s}}=0.90$ was used for $B\left(D^{+} \rightarrow \mu^{+} \nu_{\mu}\right)$.

\section{TABLE 2}

The number of $D_{s}^{+} \rightarrow \mu^{+} \nu_{\mu}$ was then extracted from a fit to the $P_{T \mu}$ distribution of the muonic KINK sample. In this fit, the background normalizations and shapes were fixed by the Monte Carlo, while the normalization for the $D_{s}^{+} \rightarrow \mu^{+} \nu_{\mu}$ decay was allowed to float. Fig. 1 a) shows the fit result for the muonic KINK decays. The points with error bars are the experimental data, while the solid, dotted and dashed histograms represent the final fit result, the sum of the backgrounds and the $D_{s}^{+} \rightarrow \mu^{+} \nu_{\mu}$ signal, respectively. The fit is in 
a very good agreement with the data, with $\chi^{2} / D . F$. is 1.029 . The fit yielded $23.2 \pm 6.0_{-0.9}^{+1.0}$ $D_{s}^{+} \rightarrow \mu^{+} \nu_{\mu}$ decays. The first error is the statistical and the second one is the systematic, involving uncertainty of production distribution. The detection efficiency for these decays is $14.6 \%$, determined mainly from a Monte Carlo calculation. It includes efficiency factors for trigger and reconstruction (0.817), offline selection cut (0.416), fraction of sample scanned (0.570), fiducial and location losses (0.956), final selection cuts (0.855), and scanning efficiency in emulsion (0.925). The overall efficiency is large and well-understood because of the simple kink topology, the weak event-selection cuts, and the high scanning efficiency. The number of the background events from $D^{+} \rightarrow \mu^{+} \nu_{\mu}$ and $D_{s}^{+} \rightarrow \tau^{+} \nu_{\tau} ; \tau^{+} \rightarrow \mu^{+} \nu_{\mu} \overline{\nu_{\tau}}$ in the high $P_{T \mu}$ region were estimated to be 4.5 and 0.28 respectively. The final background, feedthrough muons from hadronic decays, was determined to be negligible. As a systematic check, the Monte Carlo prediction for the VEE decays was compared with the data as shown in Fig. 1 b). They are in a good agreement, which gives further confidence in our analysis.

\section{FIGURE 1}

In a previous publication [9], we reported on $18.7 \pm 4.9_{-0.7}^{+0.4} D_{s}^{+} \rightarrow \phi \mu^{+} \nu_{\mu}$ events measured in E653 without emulsion information with an efficiency of 1.86\%. Combining these results, we can calculate the ratio $B\left(D_{s}^{+} \rightarrow \mu^{+} \nu_{\mu}\right) / B\left(D_{s}^{+} \rightarrow \phi \mu^{+} \nu_{\mu}\right)$ using the following equation:

$$
\frac{B\left(D_{s}^{+} \rightarrow \mu^{+} \nu_{\mu}\right)}{B\left(D_{s}^{+} \rightarrow \phi \mu^{+} \nu_{\mu}\right)}=\frac{\epsilon_{\phi \mu \nu}}{\epsilon_{\mu \nu}} \cdot \frac{N_{\mu \nu}}{N_{\phi \mu \nu}}=0.16 \pm 0.06 \pm 0.03
$$

where $\epsilon$ and $N$ are the detection efficiency and the extracted yield for each decay channel respectively. The first error is the statistical and the second one is the systematic. Using the measured value of $B\left(D_{s}^{+} \rightarrow \phi l^{+} \nu_{l}\right)=1.88 \pm 0.29 \%$ [ㅇ], we obtain,

$$
B\left(D_{s}^{+} \rightarrow \mu^{+} \nu_{\mu}\right)=(0.30 \pm 0.12 \pm 0.06 \pm 0.05) \%
$$

The first two errors are the statistical and systematic errors of our analysis, involving uncertainty of production cross section and its distribution, and the third one reflects the uncertainty in the branching fraction for $D_{s}^{+} \rightarrow \phi l^{+} \nu_{l}$. Note that this normalization does 
not require knowledge of the $D_{s}^{+}$cross section. Furthermore, it is quite insensitive to the $D_{s}^{+}$production parameters $n$ and $b$ because the muon $P_{T}$ relative to the beam direction in $D_{s}^{+} \rightarrow \mu^{+} \nu_{\mu}$ is dominated by the decay $P_{T}$ of the muon rather than by production $P_{T}$ of the $D_{s}^{+}$.

The pseudoscalar decay constant $f_{D_{s}}$ can be extracted from the following equation,

$B\left(D_{s}^{+} \rightarrow \mu^{+} \nu_{\mu}\right)=\frac{G_{F}^{2}}{8 \pi} f_{D_{s}}^{2} \tau_{D_{s}} m_{D_{s}} m_{\mu}^{2}\left|V_{c s}\right|^{2}\left(1-\frac{m_{\mu}^{2}}{m_{D_{s}}^{2}}\right)^{2}$,

where $G_{F}$ is the Fermi constant, $\tau_{D_{s}}$ and $m_{D_{s}}$ are the mean lifetime and mass of the $D_{s}^{+}$, $m_{\mu}$ is the mass of muon, and $V_{c s}$ is the Kobayashi-Maskawa matrix element. Using $\tau_{D_{s}}=$ $4.67 \pm 0.17 \times 10^{-13} s, m_{D_{s}}=1968.5 \pm 0.6 \mathrm{MeV} / \mathrm{c}^{2},\left|V_{c s}\right|=0.9745 \pm 0.0007$ [18], and our result for $B\left(D_{s}^{+} \rightarrow \mu^{+} \nu_{\mu}\right)$, we obtain,

$f_{D_{s}}=194 \pm 35 \pm 20 \pm 14 \mathrm{MeV}$

The first two errors are the statistical and systematic errors of our analysis, and the third one reflects the uncertainty in the branching fraction for $D_{s}^{+} \rightarrow \phi l^{+} \nu_{l}$.

Table 3 compares the E653 result for $f_{D_{s}}$ with those from other experiments. Agreement with WA75 [] is is excellent. Our result is also consistent at the $\sim 10 \%$ confidence level with the lager values of $f_{D_{s}}$ from CLEO [2] and BES [3]. Theoretical predictions [1, 12, 13, 14] span the range 130 to $350 \mathrm{MeV}$. Recent calculations using QCD sum rule [15], independent quark model [1], and lattice [, 17] techniques are in good agreement with our result.

\section{TABLE 3}

In conclusion, E653 has measured the ratio of the muonic branching ratios for the $D_{s}$; $B\left(D_{s}^{+} \rightarrow \mu^{+} \nu_{\mu}\right) / B\left(D_{s}^{+} \rightarrow \phi \mu^{+} \nu_{\mu}\right)=0.16 \pm 0.06 \pm 0.03$. With the latest value for $B\left(D_{s}^{+} \rightarrow\right.$ $\left.\phi \mu^{+} \nu_{\mu}\right)$, we obtain $B\left(D_{s}^{+} \rightarrow \mu^{+} \nu_{\mu}\right)=(0.30 \pm 0.12 \pm 0.06 \pm 0.05) \%$. This yields a value of $f_{D_{s}}=194 \pm 35 \pm 20 \pm 14 \mathrm{MeV}$.

We gratefully acknowledge the efforts of the Fermi National Accelerator Laboratory staff in staging this experiment. This work was supported in part by US Department of 
Energy; the US National Science Foundation; the Japan Society for the Promotion of Science; the Japan-US Cooperative Research Program for High Energy Physics; the Ministry of Education,Science and Culture of Japan; the Korea Science and Engineering Foundation; and the Basic Science Research Institute Program, Ministry of Education,Republic of Korea. 


\section{REFERENCES}

1. S. Aoki et al., Progress of Theoretical Physics Vol. 89, No. 1, pp. (1993) 131 The WA75 value was based on the $1992 \mathrm{PDG}$ value of $B\left(D_{s}^{+} \rightarrow K^{+} K^{-} \pi^{+}\right)=(3.9 \pm$ $0.4) \%$ and $B\left(D^{0} \rightarrow \mu \nu_{\mu} X\right)=(8.8 \pm 2.5) \%$. Using the new PDG [18] value of $B\left(D_{s}^{+} \rightarrow\right.$ $\left.K^{+} K^{-} \pi^{+}\right)=(4.8 \pm 0.7) \%$ and $B\left(D^{0} \rightarrow \mu \nu X\right)=(7.6 \pm 1.7) \%$, the corrected value is $f_{D_{s}}=(238 \pm 47 \pm 21 \pm 43) M e V / c^{2}$.

2. D. Acosta et al., Phys. Rev. D 49 (1994) 5690.

3. J.Z. Bai et al., Phys. Rev. Lett. 74 (1995) 4599.

4. H. Albrecht et al., Z. Phys. C 54 (1992) 1.

5. K. Kodama et al., Nucl. Instr. \& Meth. A 289 (1990) 146.

6. K. Kodama et al., Nucl. Instr. \& Meth. B 93 (1994) 340.

7. C.W. Bernard, J.N. Labrenz, A. Soni, Phys. Rev. D 49 (1994) 2536.

8. K. Kodama et al., Phys. Lett. B 284 (1992) 461.

9. K. Kodama et al., Phys. Lett. B 309 (1993) 483.

10. S. Barlag et al., Phys. Lett. B 247 (1990) 113.

K. Kodama et al., Phys. Lett. B 286 (1992) 187.

11. M. Suzuki, Phys. Lett. B 162 (1985) 392.

12. S.N. Sinha, Phys. Lett. B 178 (1986) 110.

13. S. Narison, Phys. Lett. B 198 (1987) 104.

14. P. Colangelo, G. Nardulli, and M. Pietroni, Phys. Rev. D 43 (1991) 3002.

15. K. Schilcher, Y.L. Wu, Z. Phys. C 54 (1992) 163.

16. N. Barik, P.C. Dash, Phys. Rev. D 47 (1993) 2788. 
17. C. Bernard et al., Nucl. Phys. B, Proc. Suppl. 42 (1995) 388.

18. L. Montanet et al., Phys. Rev. D 50, 1173 (1994) and 1995 off-year partial update for the 1996 edition (URL:http://pdg.lbl.gov/) 


\section{FIGURES}

Fig. 1. Decay $P_{T \mu}$ distributions of a) 531 KINK (muonic one prong) events, and b) 276 VEE (muonic two prong) events. The points with error bars are the experimental data, and the histograms are the Monte Carlo simulations. The vertical dotted lines show the decay $P_{T \mu}$ limits of the main semi-leptonic modes. In (a), the solid, dotted and dashed histograms represent the final

fit result, the sum of the backgrounds, and the $D_{s}^{+} \rightarrow \mu^{+} \nu_{\mu}$ signal, respectively. In (b), the solid line represents the inclusive spectrum of muons from $D^{0}$ decay. 


\section{TABLES}

Table 1. Summary of offline selection criterion and cut requirements.

\begin{tabular}{ll}
\hline Offline selection & $P_{T \mu \text { Beam }}>0.8 \mathrm{GeV} / \mathrm{c}$ \\
Flight length cut & $F L>500 \mu \mathrm{m}$ \\
Decay $P_{T \mu}$ cut & $P_{T \mu}>0.28 \mathrm{GeV} / \mathrm{c}$ \\
Vertex position cut(only for VEE) & Decay vertex in emulsion bulk \\
\hline \hline
\end{tabular}

Table 2. Cross sections used for Monte Carlo background calculations.

\begin{tabular}{lc}
\hline \hline$\sigma\left(D^{ \pm} ; x_{F}>0\right)$ & $8.66 \pm 0.46 \pm 1.96 \mu b /$ nucleon $[$ 目] \\
$\sigma\left(D^{0} ; x_{F}>0\right)$ & $22.05 \pm 1.37 \pm 4.82 \mu b /$ nucleon [8] \\
$\sigma\left(D_{s}^{ \pm} ; x_{F}>0\right)$ & $5.1 \pm 1.3 \pm 1.3 \mu b /$ nucleon [8, 18$]$ \\
$\sigma\left(\Lambda_{c}^{ \pm} ; x_{F}>0\right)$ & $15.3 \pm 1.7 \pm 2.6 \mu b /$ nucleon $[1]$ ] \\
\hline \hline
\end{tabular}

Table 3. Summary of published measurement of pseudoscalar decay constant $f_{D_{s}}$.

\begin{tabular}{|c|c|}
\hline Experiment & $f_{D_{s}}(M e V)$ \\
\hline E653 (This work) & $194 \pm 35 \pm 20 \pm 14$ \\
\hline WA75 凅 (1993) & $238 \pm 47 \pm 21 \pm 43$ \\
\hline CLEO [2] (1994) & $344 \pm 37 \pm 52 \pm 42$ \\
\hline BES [ [ (1995) & $430_{-130}^{+150} \pm 40$ \\
\hline
\end{tabular}


DPNU-96-33

June 1996

Measurement of $B\left(D_{s}^{+} \rightarrow \mu^{+} \nu_{\mu}\right) / B\left(D_{s}^{+} \rightarrow \phi \mu^{+} \nu_{\mu}\right)$ and Determination of the Decay Constant $f_{D_{s}}$

Fermilab E653 Collaboration

K. Kodama ${ }^{(1)}$, S. Torikai ${ }^{(1)}$, N. Ushida ${ }^{(1)}$, A. Mokhtarani ${ }^{(2),(a)}$, V.S. Paolone ${ }^{(2)}$, J.T. Volk ${ }^{(2),(a)}$, J.O. Wilcox ${ }^{(2),(b)}$, P.M. Yager ${ }^{(2)}$, R.M. Edelstein ${ }^{(3)}$, A.P. Freyberger ${ }^{(3),(c)}$,

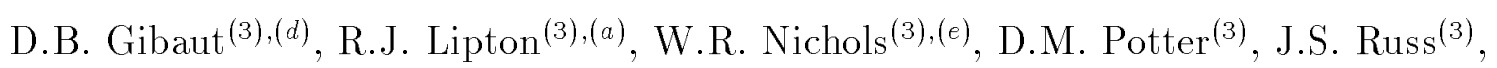
C. Zhang ${ }^{(3)}$, Y. Zhang ${ }^{(3),(f)}$, H.I. Jang ${ }^{(4)}$, J.Y. Kim ${ }^{(4)}$, B.R. Baller ${ }^{(5)}$, R.J. Stefanski ${ }^{(5)}$, K. Nakazawa ${ }^{(6)}$, S.H. Chung( ${ }^{(7)}$, M.S. Park ${ }^{(7)}$, I.G. Park ${ }^{(7)}$, J.S. Song( ${ }^{(7)}$, C.S. Yoon ${ }^{(7)}$,

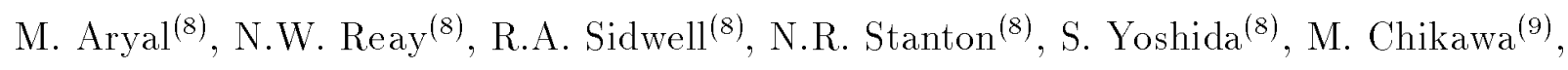

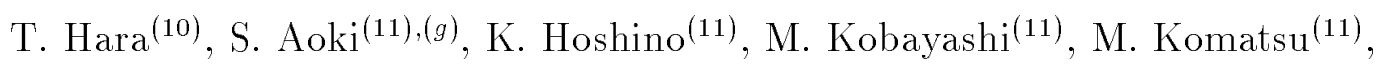

M. Miyanishi(11), M. Nakamura ${ }^{(11)}$, S. Nakanishi(11), K. Niwa ${ }^{(11)}$, M. Nomura ${ }^{(11)}$,

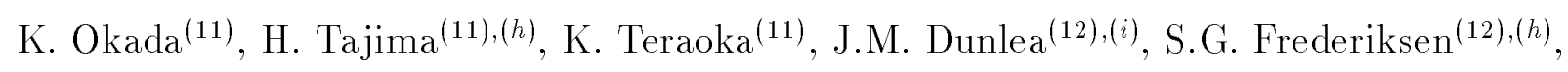
S. Kuramata ${ }^{(12),(j)}$, B.G. Lundberg ${ }^{(12),(a)}$, G.A. Oleynik ${ }^{(12),(a)}$, K. Reibel ${ }^{(12)}$, G.R. Kalbfleisch ${ }^{(13)}$, P. Skubic ${ }^{(13)}$, J.M. Snow ${ }^{(13)}$, S.E. Willis ${ }^{(13),(k)}$, K. Nakamura ${ }^{(14)}$, T. Okusawa ${ }^{(14)}$, T. Yoshida ${ }^{(14)}$, H. Okabe ${ }^{(15)}$, J. Yokota ${ }^{(15)}$, N. Ihara ${ }^{(16)}$, M. Kazuno $^{(16)}$,

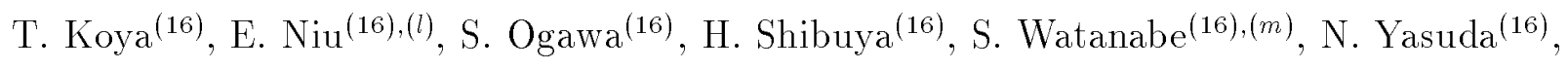
K. Ehara ${ }^{(17)}$, K. Horie ${ }^{(17)}$, Y. Sato ${ }^{(17)}$, K. Suzuki ${ }^{(17)}$, and I. Tezuka ${ }^{(17)}$

\begin{abstract}
We have observed 23.2 $26.0_{-0.9}^{+1.0}$ purely-leptonic decays of $D_{s}^{+} \rightarrow \mu^{+} \nu_{\mu}$ from a sample of muonic one prong decay events detected in the emulsion target of Fermilab experiment E653. Using the $D_{s}^{+} \rightarrow \phi \mu^{+} \nu_{\mu}$ yield measured previously in this experiment, we obtain $B\left(D_{s}^{+} \rightarrow \mu^{+} \nu_{\mu}\right) / B\left(D_{s}^{+} \rightarrow \phi \mu^{+} \nu_{\mu}\right)=0.16 \pm 0.06 \pm 0.03$. In addition, we extract the decay constant $f_{D_{s}}=194 \pm 35 \pm 20 \pm 14 \mathrm{MeV}$.
\end{abstract}


1) Aichi University of Education, Kariya 448, JAPAN

2) University of California (Davis), Davis, CA 95616, USA

3) Carnegie-Mellon University, Pittsburgh, PA 15213, USA

4) Chonnam National University, Kwangju 500-757, KOREA

5) Fermi National Accelerator Laboratory, Batavia, IL 60510, USA

6) Gifu University, Gifu 501-11, JAPAN

7) Gyeongsang National University, Jinju 660-300, KOREA

8) Kansas State University, Manhattan, KS 66506, USA

9) Kinki University, Higashi-Osaka 577, JAPAN

10) Kobe University, Kobe 657, JAPAN

11) Nagoya University, Nagoya 464, JAPAN

12) The Ohio State University, Columbus, OH 43210, USA

13) University of Oklahoma, Norman, OK 73019, USA

14) Osaka City University, Osaka 558, JAPAN

15) Science Education Institute of Osaka Prefecture, Osaka 558, JAPAN

16) Toho University, Funabashi 274, JAPAN

17) Utsunomiya University, Utsunomiya 321, JAPAN

${ }^{a}$ Fermilab, Batavia, IL 60510, USA.

${ }^{b}$ Northeastern University, Boston, MA 02115, USA.

${ }^{c}$ CEBAF, 12000 Jefferson Avenue Newport News, VA 23606, USA.

${ }^{d}$ Virginia Polytechnic Institute and State University, Blacksburg, VA 24060, USA.

${ }^{e}$ Westinghouse Electric Corp., Pittsburgh, PA 15230, USA.

${ }^{f}$ Pennsylvania State University, University Park, PA 16802, USA.

${ }^{g}$ Kobe University, Kobe 657, JAPAN.

${ }^{h}$ University of Tokyo 7-3-1 Hongo, Bunkyou-ku, Tokyo 113 JAPAN.

${ }^{i}$ University of Rochester, Rochester, NY 14627, USA.

${ }^{j}$ Hirosaki University, Hirosaki 036, JAPAN.

${ }^{k}$ Northern Illinois University, DeKalb, IL 60115, USA. 
${ }^{l}$ CERN, CH-1211, Geneva, SWITZERLAND.

${ }^{m}$ University of Wisconsin, Madison, WI 53706, USA. 
Leptonic decay of charged pseudoscalar mesons is described by the annihilation of constituent quark and antiquark into a virtual $W$ boson. The rate for this process is proportional to the square of the pseudoscalar decay constant $f$, which measures the overlap of quark and antiquark at zero separation. The constant $f$ gives absolute normalizations of numerous heavy-flavor transitions, including mixing, semi-leptonic and non-leptonic decays. Hence it is highly desirable to determine the pseudoscalar decay constant more precisely in charm and $B$ physics. A measurement of a purely-leptonic decay branching ratio, unlike semi-leptonic or non-leptonic decays, is the most reliable way to extract the pseudoscalar decay constant, because it does not involve any QCD corrections.

There are three purely-leptonic decay modes for the $D_{s}^{+}$(charge conjugate modes are implied throughout this paper): electronic, muonic and tauonic. Among them, the muonic decay is the most accessible mode to determine the decay constant $f_{D_{s}}$. The electronic decay is helicity suppressed by five orders of magnitude with respect to the muonic one. While the tauonic decay branching ratio is expected to be an order of magnitude larger than the muonic decay, the $\mathrm{Q}$ value for $D_{s}^{+} \rightarrow \tau^{+} \nu_{\tau}$ is small and hence reconstruction of this mode is extremely difficult because of the small decay angle in the lab frame.

The first measurement of the pseudoscalar decay constant of the charmed strange meson, $f_{D_{s}}$, was carried out by the WA75 group in $1993\left[{ }^{1}\right]$, using a transverse momentum spectrum of muons from $D_{s}^{+}$leptonic decays observed in an emulsion target. They obtained a value of $f_{D_{s}}=(232 \pm 45 \pm 20 \pm 48) \mathrm{MeV}$. CLEO [ [ $\left.{ }^{2}\right]$ and BES [3] also reported recent measurements of $f_{D_{s}}$, and ARGUS [ $\left.{ }^{4}\right]$ obtained $f_{D_{s}}$ by model dependent calculation in 1992. All of these results, however, suffer from large statistical and systematic errors, and hence the present measured value of $f_{D_{s}}$ is still not determined well enough to discriminate between different theoretical models. In this paper, we report an independent measurement of $f_{D_{s}}$ from an analysis of the data from Fermilab experiment E653.

The Fermilab emulsion-hybrid experiment E653 was designed to study production and decay of heavy flavor particles by the direct observation of decay vertices in the emulsion. Data for this analysis were taken in a $600 \mathrm{GeV} / \mathrm{c} \pi^{-}$beam during the second run of Fermilab 
E653. The trigger required both a beam particle to interact in the target and a muon to penetrate $3,900 \mathrm{~g} / \mathrm{cm}^{2}$ of absorber. This muon trigger provided an enriched sample of semi-muonic decays of heavy flavor particles. A total of $8.2 \times 10^{6}$ events, corresponding to $2.5 \times 10^{8}$ interactions, were recorded during this run. Since the details of this experiment were described in another paper [5], only a brief description of each detector element is given here.

The detector consisted of the emulsion target, tracking detector, spectrometer, and muon detection system. Because of its unsurpassed spatial resolution, nuclear emulsion was chosen as an active target, and it was used both as a primary interaction target and as a decay vertex detector. The electronic detectors downstream of the target select events for the emulsion scanning and predict the interaction vertices and each track position in the emulsion. Tracks from interactions and decays in the emulsion were measured in the vertex silicon strip detectors (VSSDs). The VSSDs consisted of 18 planes arranged in 6 groups of three readout coordinates, and the center part of the detector achieved a resolution transverse to the beam of $8.8 \mu \mathrm{m}$. This detector also served as the upstream arm of the spectrometer. The spectrometer gave a momentum resolution of $\sigma_{p} / p=\sqrt{(0.01)^{2}+(0.00023 p)^{2}} \quad(p$ in $G e V / c)$. The most down stream element of the E653 detector array was the muon identification system, which remeasured the direction and momenta of muon candidates after the absorber with drift chambers and an iron toroid.

To enrich the relative yield of events with heavy flavor particle decays and to reduce the emulsion scanning load, the transverse momentum of the triggered muon with respect to the beam axis, $P_{T \mu \text { Beam }}$, was required to be greater than $0.8 \mathrm{GeV} / \mathrm{c}$. In total, 94,000 events passed this criterion from a initial sample of $8.2 \times 10^{6}$ events. This reduced sample contained a significant number of background events from feedthrough muons. Pions or kaons could be misidentified as muons because they decayed muonically in flight with small angle deflections or from punch through in the iron absorber. These events were rejected using the method described below.

In the emulsion analysis, the primary interaction vertices were visually located in the 
emulsion by a semi-automatic scanning microscope system using position predictions from the VSSDs. The efficiency for finding primary vertices was determined to be greater than $99 \%$. If the angle of an emulsion track from the primary vertex matched to within 1 mrad of the reconstructed muon track, the muon was identified as a feedthrough muon and the event was rejected. For the remaining events, a more detailed emulsion scanning technique was employed to find the origin of the muon.

Different decay search methods were applied for charged and neutral decay vertices. To find charged decays, all emulsion tracks observed at the primary vertex which did not match to any track reconstructed by the VSSDs were followed towards the most downstream emulsion plates. To find neutral decays, the tracks reconstructed by the VSSDs with no corresponding emulsion track observed at the primary vertex, were located at the most downstream plate of the emulsion target. Once found, these tracks were followed upstream through the emulsion stack to their origin within the emulsion target to detect neutral decays. The detailed event location and vertex search methods are described elsewhere $\left.{ }^{6}\right]$.

As a result of the above analysis, 1,193 charmed particle candidates were identified: 565 KINK (charged one prong) decays, 536 VEE (neutral two prong) decays, and 92 three or four prong decays. Further cuts on the decay length $(>500 \mu \mathrm{m})$ and the transverse momentum of the muon with respect to the parent particle direction $\left(P_{T \mu}>0.28 \mathrm{GeV} / \mathrm{c}\right)$ were applied to the KINK and VEE decays to improve position resolution measurement and to reject strange particle decays. Only VEEs with their vertices found in the emulsion were retained to avoid the large $P_{T \mu}$ error due to the intrinsically larger position errors of the VSSDs. The offline selection criteria and cuts are summarized in Table 1.

\section{TABLE 1}

Figure 1 shows the $P_{T \mu}$ distributions for 531 KINKs and 276 VEEs which survived the above selection criteria. An excess of 19 events can be seen in the region $\left(P_{T \mu}>0.865 \mathrm{GeV} / \mathrm{c}\right)$ exceeding the kinematic limit of the $D^{+} \rightarrow \bar{K}^{0} \mu^{+} \nu_{\mu}$ decay for the KINK events, while no such signal is seen for the VEE events. These large $P_{T \mu}$ events can be interpreted as the 
fully leptonic decay $D_{s}^{+} \rightarrow \mu^{+} \nu_{\mu}$.

In addition to $D_{s}^{+} \rightarrow \mu^{+} \nu_{\mu}$ this sample includes background events. The largest source of background is the muon from the decay $D^{+} \rightarrow \mu^{+} \nu_{\mu}$. The inclusive production cross section for $D^{+}$is a factor of two larger than $D_{s}$. However, the branching fraction for this decay is considerably smaller than $D_{s}^{+} \rightarrow \mu^{+} \nu_{\mu}$, because it is suppressed by the ratio of CKM matrixelement, $\left|V_{c d}\right|^{2} /\left|V_{c s}\right|^{2}$. A muon from the cascade decay, $D_{s}^{+} \rightarrow \tau^{+} \nu_{\tau} ; \tau^{+} \rightarrow \mu^{+} \nu_{\mu} \overline{\nu_{\tau}}$ is another source of background. Since the decay angle of the first decay point is extremely small ( $\approx 1-2 \mathrm{mrad})$, it is often not detectable by our ordinary emulsion scanning method $\left[^{6}\right.$. Therefore, only the second decay $\tau^{+} \rightarrow \mu^{+} \nu_{\mu} \overline{\nu_{\tau}}$ is observable and therefore will appear in the emulsion as a muonic KINK. A final possible background is a feedthrough muon from the daughter track of a hadronic charm decay.

We used a Monte Carlo simulation to estimate the total number of background events. Charged and neutral charmed particles were generated, using the production distribution parametrization $d^{2} \sigma / d x_{F} d P_{T}^{2} \propto\left(1-\left|x_{F}\right|\right)^{n} \cdot \exp \left(-b P_{T}^{2}\right)$ with $n=4.25 \pm 0.24 \pm 0.23$ and $b=0.76 \pm 0.03 \pm 0.03(\mathrm{GeV} / \mathrm{c})^{-2}\left[^{8}\right]$. The relative numbers of $D^{+}, D^{0}$ and $D_{s}^{+}$mesons were generated according to the the cross section values measured in this experiment $\left[{ }^{8},{ }^{9},{ }^{18}\right]$. The baryon $\Lambda_{c}^{+}$was generated using the cross section value obtained from other experiments [10]. The cross section numbers employed in the Monte Carlo are summarized in Table 2. Decays of charmed particles were simulated by JETSET 7.4 with known branching fractions [ $\left.{ }^{18}\right]$, except that the ratio $f_{D^{+}} / f_{D_{s}}=0.90\left[{ }^{7}\right]$ was used for $B\left(D^{+} \rightarrow \mu^{+} \nu_{\mu}\right)$.

\section{TABLE 2}

The number of $D_{s}^{+} \rightarrow \mu^{+} \nu_{\mu}$ was then extracted from a fit to the $P_{T \mu}$ distribution of the muonic KINK sample. In this fit, the background normalizations and shapes were fixed by the Monte Carlo, while the normalization for the $D_{s}^{+} \rightarrow \mu^{+} \nu_{\mu}$ decay was allowed to float. Fig. 1 a) shows the fit result for the muonic KINK decays. The points with error bars are the experimental data, while the solid, dotted and dashed histograms represent the final fit result, the sum of the backgrounds and the $D_{s}^{+} \rightarrow \mu^{+} \nu_{\mu}$ signal, respectively. The fit is in a very good 
agreement with the data, with $\chi^{2} / D . F$. is 1.029 . The fit yielded $23.2 \pm 6.0_{-0.9}^{+1.0} D_{s}^{+} \rightarrow \mu^{+} \nu_{\mu}$ decays. The first error is the statistical and the second one is the systematic, involving uncertainty of production distribution. The detection efficiency for these decays is $14.6 \%$, determined mainly from a Monte Carlo calculation. It includes efficiency factors for trigger and reconstruction (0.817), offline selection cut (0.416), fraction of sample scanned $(0.570)$, fiducial and location losses (0.956), final selection cuts (0.855), and scanning efficiency in emulsion (0.925). The overall efficiency is large and well-understood because of the simple kink topology, the weak event-selection cuts, and the high scanning efficiency. The number of the background events from $D^{+} \rightarrow \mu^{+} \nu_{\mu}$ and $D_{s}^{+} \rightarrow \tau^{+} \nu_{\tau} ; \tau^{+} \rightarrow \mu^{+} \nu_{\mu} \overline{\nu_{\tau}}$ in the high $P_{T \mu}$ region were estimated to be 4.5 and 0.28 respectively. The final background, feedthrough muons from hadronic decays, was determined to be negligible. As a systematic check, the Monte Carlo prediction for the VEE decays was compared with the data as shown in Fig. 1 b). They are in a good agreement, which gives further confidence in our analysis.

\section{FIGURE 1}

In a previous publication [9], we reported on $18.7 \pm 4.9_{-0.7}^{+0.4} D_{s}^{+} \rightarrow \phi \mu^{+} \nu_{\mu}$ events measured in E653 without emulsion information with an efficiency of $1.86 \%$. Combining these results, we can calculate the ratio $B\left(D_{s}^{+} \rightarrow \mu^{+} \nu_{\mu}\right) / B\left(D_{s}^{+} \rightarrow \phi \mu^{+} \nu_{\mu}\right)$ using the following equation:

$$
\frac{B\left(D_{s}^{+} \rightarrow \mu^{+} \nu_{\mu}\right)}{B\left(D_{s}^{+} \rightarrow \phi \mu^{+} \nu_{\mu}\right)}=\frac{\epsilon_{\phi \mu \nu}}{\epsilon_{\mu \nu}} \cdot \frac{N_{\mu \nu}}{N_{\phi \mu \nu}}=0.16 \pm 0.06 \pm 0.03
$$

where $\epsilon$ and $N$ are the detection efficiency and the extracted yield for each decay channel respectively. The first error is the statistical and the second one is the systematic. Using the measured value of $B\left(D_{s}^{+} \rightarrow \phi l^{+} \nu_{l}\right)=1.88 \pm 0.29 \%\left[{ }^{18}\right]$, we obtain,

$$
B\left(D_{s}^{+} \rightarrow \mu^{+} \nu_{\mu}\right)=(0.30 \pm 0.12 \pm 0.06 \pm 0.05) \%
$$

The first two errors are the statistical and systematic errors of our analysis, involving uncertainty of production cross section and its distribution, and the third one reflects the uncertainty in the branching fraction for $D_{s}^{+} \rightarrow \phi l^{+} \nu_{l}$. Note that this normalization does 
not require knowledge of the $D_{s}^{+}$cross section. Furthermore, it is quite insensitive to the $D_{s}^{+}$production parameters $n$ and $b$ because the muon $P_{T}$ relative to the beam direction in $D_{s}^{+} \rightarrow \mu^{+} \nu_{\mu}$ is dominated by the decay $P_{T}$ of the muon rather than by production $P_{T}$ of the $D_{s}^{+}$.

The pseudoscalar decay constant $f_{D_{s}}$ can be extracted from the following equation,

$$
B\left(D_{s}^{+} \rightarrow \mu^{+} \nu_{\mu}\right)=\frac{G_{F}^{2}}{8 \pi} f_{D_{s}}^{2} \tau_{D_{s}} m_{D_{s}} m_{\mu}^{2}\left|V_{c s}\right|^{2}\left(1-\frac{m_{\mu}^{2}}{m_{D_{s}}^{2}}\right)^{2},
$$

where $G_{F}$ is the Fermi constant, $\tau_{D_{s}}$ and $m_{D_{s}}$ are the mean lifetime and mass of the $D_{s}^{+}$, $m_{\mu}$ is the mass of muon, and $V_{c s}$ is the Kobayashi-Maskawa matrix element. Using $\tau_{D_{s}}=$ $4.67 \pm 0.17 \times 10^{-13} s, m_{D_{s}}=1968.5 \pm 0.6 \mathrm{MeV} / \mathrm{c}^{2},\left|V_{c s}\right|=0.9745 \pm 0.0007\left[{ }^{18}\right]$, and our result for $B\left(D_{s}^{+} \rightarrow \mu^{+} \nu_{\mu}\right)$, we obtain,

$f_{D_{s}}=194 \pm 35 \pm 20 \pm 14 \mathrm{MeV}$

The first two errors are the statistical and systematic errors of our analysis, and the third one reflects the uncertainty in the branching fraction for $D_{s}^{+} \rightarrow \phi l^{+} \nu_{l}$.

Table 3 compares the E653 result for $f_{D_{s}}$ with those from other experiments. Agreement with WA75 $\left.{ }^{1}\right]$ is excellent. Our result is also consistent at the $\sim 10 \%$ confidence level with the lager values of $f_{D_{s}}$ from CLEO $\left[{ }^{2}\right]$ and BES [3]. Theoretical predictions $\left[{ }^{11},{ }^{12},{ }^{13},{ }^{14}\right]$ span the range 130 to $350 \mathrm{MeV}$. Recent calculations using QCD sum rule [15], independent quark model $\left[{ }^{16}\right]$, and lattice $\left[{ }^{7}{ }^{17}\right]$ techniques are in good agreement with our result.

\section{TABLE 3}

In conclusion, E653 has measured the ratio of the muonic branching ratios for the $D_{s}$; $B\left(D_{s}^{+} \rightarrow \mu^{+} \nu_{\mu}\right) / B\left(D_{s}^{+} \rightarrow \phi \mu^{+} \nu_{\mu}\right)=0.16 \pm 0.06 \pm 0.03$. With the latest value for $B\left(D_{s}^{+} \rightarrow\right.$ $\left.\phi \mu^{+} \nu_{\mu}\right)$, we obtain $B\left(D_{s}^{+} \rightarrow \mu^{+} \nu_{\mu}\right)=(0.30 \pm 0.12 \pm 0.06 \pm 0.05) \%$. This yields a value of $f_{D_{s}}=194 \pm 35 \pm 20 \pm 14 \mathrm{MeV}$.

We gratefully acknowledge the efforts of the Fermi National Accelerator Laboratory staff in staging this experiment. This work was supported in part by US Department of Energy; 
the US National Science Foundation; the Japan Society for the Promotion of Science; the Japan-US Cooperative Research Program for High Energy Physics; the Ministry of Education,Science and Culture of Japan; the Korea Science and Engineering Foundation; and the Basic Science Research Institute Program, Ministry of Education,Republic of Korea. 


\section{REFERENCES}

1. S. Aoki et al., Progress of Theoretical Physics Vol. 89, No. 1, pp. (1993) 131 The WA75 value was based on the $1992 \mathrm{PDG}$ value of $B\left(D_{s}^{+} \rightarrow K^{+} K^{-} \pi^{+}\right)=(3.9 \pm$ $0.4) \%$ and $B\left(D^{0} \rightarrow \mu \nu_{\mu} X\right)=(8.8 \pm 2.5) \%$. Using the new PDG [18] value of $B\left(D_{s}^{+} \rightarrow\right.$ $\left.K^{+} K^{-} \pi^{+}\right)=(4.8 \pm 0.7) \%$ and $B\left(D^{0} \rightarrow \mu \nu X\right)=(7.6 \pm 1.7) \%$, the corrected value is $f_{D_{s}}=(238 \pm 47 \pm 21 \pm 43) \mathrm{MeV} / \mathrm{c}^{2}$.

2. D. Acosta et al., Phys. Rev. D 49 (1994) 5690.

3. J.Z. Bai et al., Phys. Rev. Lett. 74 (1995) 4599.

4. H. Albrecht et al., Z. Phys. C 54 (1992) 1.

5. K. Kodama et al., Nucl. Instr. \& Meth. A 289 (1990) 146.

6. K. Kodama et al., Nucl. Instr. \& Meth. B 93 (1994) 340.

7. C.W. Bernard, J.N. Labrenz, A. Soni, Phys. Rev. D 49 (1994) 2536.

8. K. Kodama et al., Phys. Lett. B 284 (1992) 461.

9. K. Kodama et al., Phys. Lett. B 309 (1993) 483.

10. S. Barlag et al., Phys. Lett. B 247 (1990) 113.

K. Kodama et al., Phys. Lett. B 286 (1992) 187.

11. M. Suzuki, Phys. Lett. B 162 (1985) 392.

12. S.N. Sinha, Phys. Lett. B 178 (1986) 110.

13. S. Narison, Phys. Lett. B 198 (1987) 104.

14. P. Colangelo, G. Nardulli, and M. Pietroni, Phys. Rev. D 43 (1991) 3002.

15. K. Schilcher, Y.L. Wu, Z. Phys. C 54 (1992) 163.

16. N. Barik, P.C. Dash, Phys. Rev. D 47 (1993) 2788. 
17. C. Bernard et al., Nucl. Phys. B, Proc. Suppl. 42 (1995) 388.

18. L. Montanet et al., Phys. Rev. D 50, 1173 (1994) and 1995 off-year partial update for the 1996 edition (URL:http://pdg.lbl.gov/) 


\section{FIGURES}

Fig. 1. Decay $P_{T \mu}$ distributions of a) 531 KINK (muonic one prong) events, and b) 276 VEE (muonic two prong) events. The points with error bars are the experimental data, and the histograms are the Monte Carlo simulations. The vertical dotted lines show the decay $P_{T \mu}$ limits of the main semi-leptonic modes. In (a), the solid, dotted and dashed histograms represent the final fit result, the sum of the backgrounds, and the $D_{s}^{+} \rightarrow \mu^{+} \nu_{\mu}$ signal, respectively. In (b), the solid line represents the inclusive spectrum of muons from $D^{0}$ decay. 


\section{TABLES}

Table 1. Summary of offline selection criterion and cut requirements.

Offline selection

Flight length cut

Decay $P_{T \mu}$ cut

Vertex position cut(only for VEE)
$P_{T \mu \text { Beam }}>0.8 \mathrm{GeV} / \mathrm{c}$

$F L>500 \mu m$

$P_{T \mu}>0.28 \mathrm{GeV} / \mathrm{c}$

Decay vertex in emulsion bulk

Table 2. Cross sections used for Monte Carlo background calculations.

\begin{tabular}{ll}
\hline \hline$\sigma\left(D^{ \pm} ; x_{F}>0\right)$ & $8.66 \pm 0.46 \pm 1.96 \mu b /$ nucleon $\left.{ }^{8}\right]$ \\
$\sigma\left(D^{0} ; x_{F}>0\right)$ & $22.05 \pm 1.37 \pm 4.82 \mu b /$ nucleon $\left.{ }^{8}\right]$ \\
$\sigma\left(D_{s}^{ \pm} ; x_{F}>0\right)$ & $5.1 \pm 1.3 \pm 1.3 \mu b /$ nucleon $\left[{ }^{9},{ }^{18}\right]$ \\
$\sigma\left(\Lambda_{c}^{ \pm} ; x_{F}>0\right)$ & $15.3 \pm 1.7 \pm 2.6 \mu b /$ nucleon $\left[{ }^{10}\right]$ \\
\hline \hline
\end{tabular}

Table 3. Summary of published measurement of pseudoscalar decay constant $f_{D_{s}}$.

\begin{tabular}{lc}
\hline \hline Experiment & $f_{D_{s}}(\mathrm{MeV})$ \\
\hline E653 (This work) & $194 \pm 35 \pm 20 \pm 14$ \\
WA75 $\left.{ }^{1}\right](1993)$ & $238 \pm 47 \pm 21 \pm 43$ \\
CLEO $\left.{ }^{2}\right](1994)$ & $344 \pm 37 \pm 52 \pm 42$ \\
BES [ $\left.{ }^{3}\right](1995)$ & $430_{-130}^{+150} \pm 40$ \\
\hline \hline
\end{tabular}



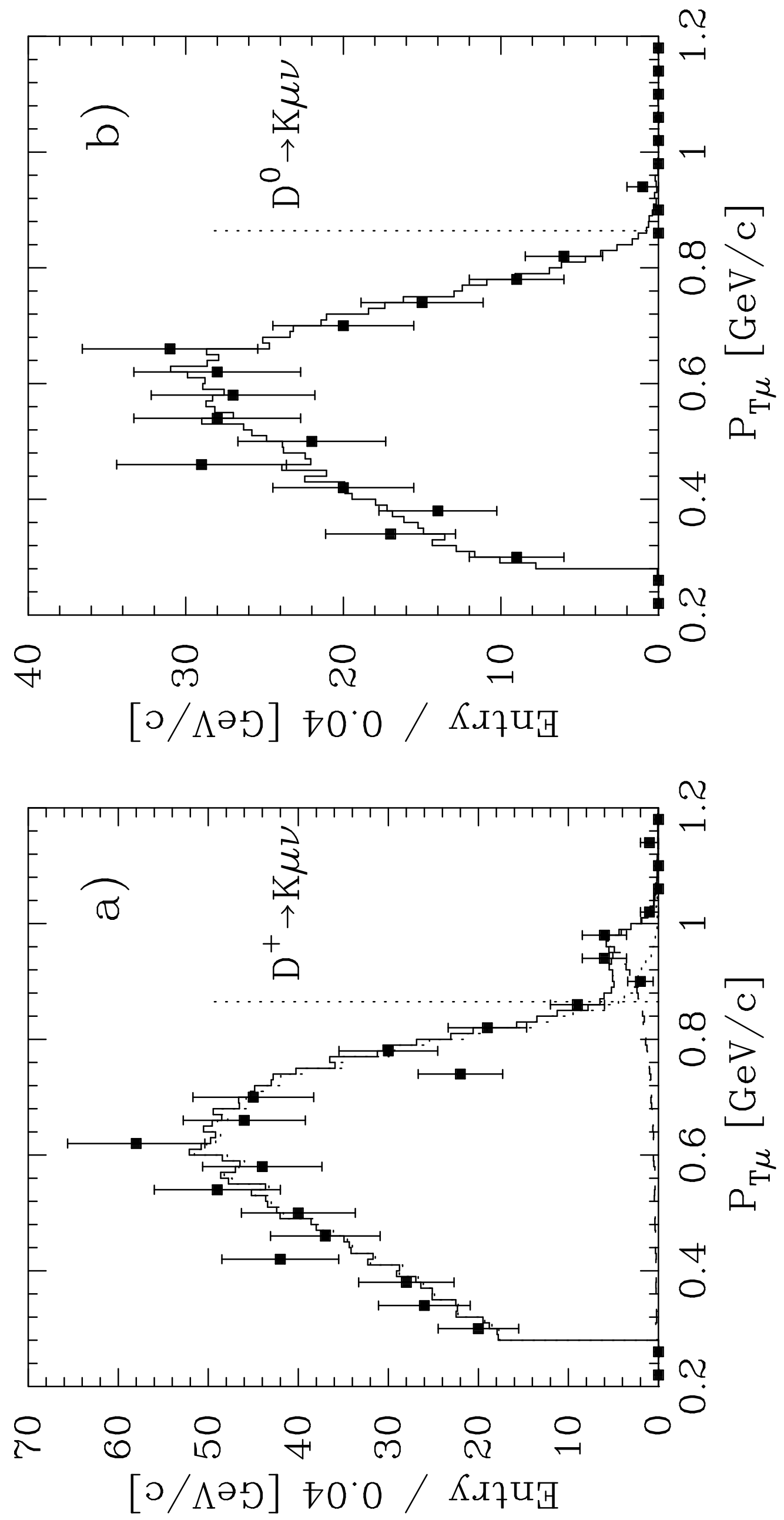

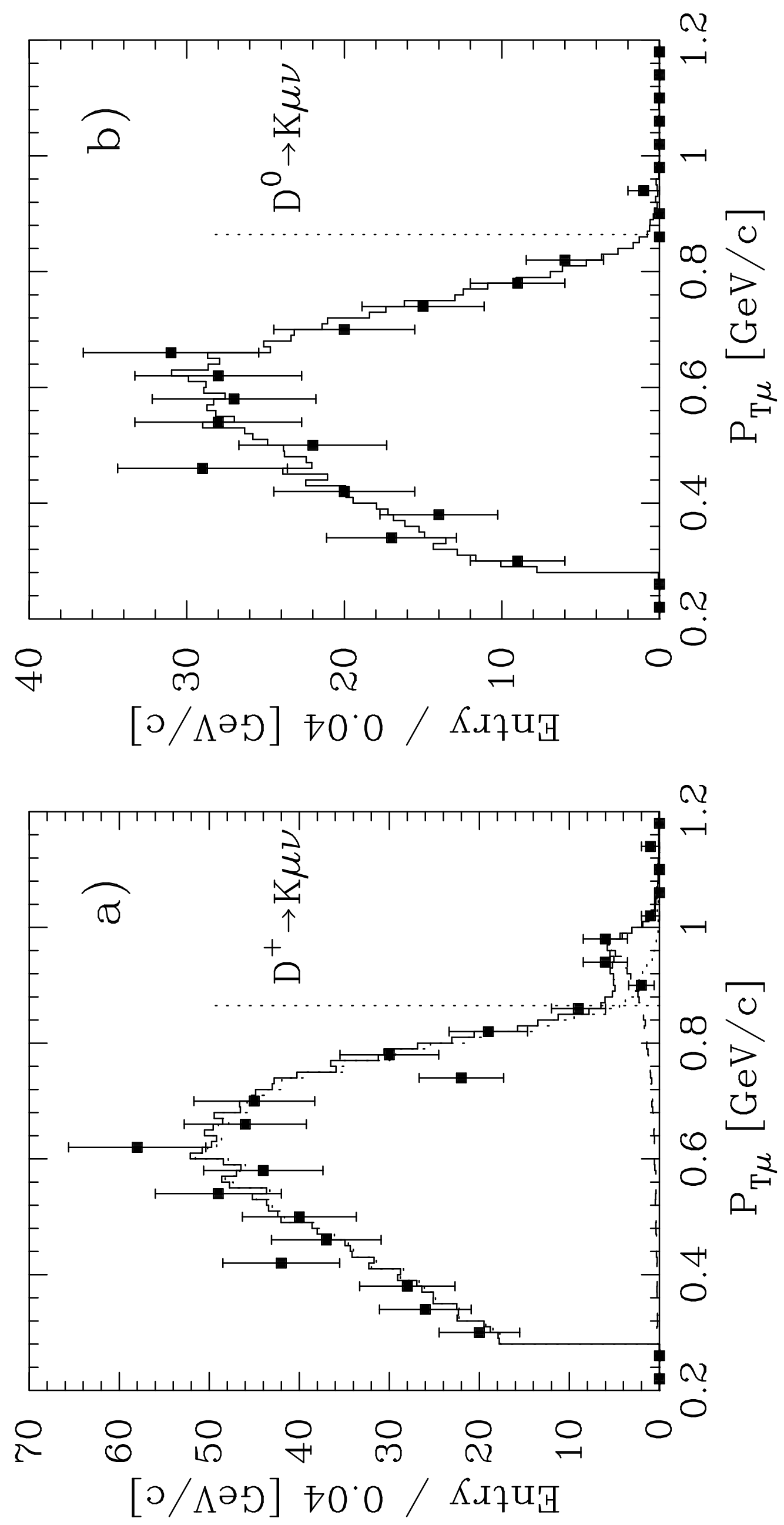